\title{
2. COMETARY OBSERVATIONS AND VARIATIONS IN COMETARY BRIGHTNESS
}

\author{
S. K. VSEKHSVYATSKIJ \\ Astronomical Observatory, Kiev University, Kiev, U.S.S.R.
}

\begin{abstract}
The cometary observation service is surveyed. Absolute magnitudes and other physical characteristics are given for the comets that appeared during 1965-1970. The general laws of cometary brightness, in particular the secular decrease in brightness, are discussed. Remarks are made about the standardization of cometary photometry.
\end{abstract}

The establishment of the cometary observation service, undertaken in the International Quiet Sun Year (IQSY) and afterwards, led to a considerable increase both in the number of observations and in the number of observatories participating in these observations. The expansion of cometary studies was due, first of all, to the ephemerides that were calculated, determinations of orbits and the dissemination of information being accomplished mainly by the International Astronomical Union's Central Bureau for Astronomical Telegrams (B. G. Marsden), as well as by the Comet and Computing Sections of the British Astronomical Association (S. W. Milbourn, C. Dinwoodie), Japanese and Czechoslovak astronomers, the Comet Centre in Kiev and the Institute for Theoretical Astronomy in Leningrad.

As for the determination of positions, it is necessary to mention the outstanding contribution of the American astronomers, who during recent years have developed an efficient system for observation of both new and periodic, and especially the faint, comets, using for this purpose large telescopes and other modern devices (G. Van Biesbroeck, E. Roemer, Smithsonian satellite-tracking network).

The international comet service acknowledges also the numerous series of observations made by the astronomers from Tokyo and elsewhere in Japan (K. Tomita, T. Seki and others), the outstanding successes of the Japanese discoverers of new comets, the observations at the Skalnaté Pleso and Klet Observatories (A. Mrkos, M. Antal), in Nice (B. Milet), at the Crimean Astrophysical Observatory (N. S. Chernykh), and in the Southern Hemisphere, with observations in Córdoba (Z. M. Pereyra), Santiago (C. Torres), Bickley (M. P. Candy), and elsewhere.

In the U.S.S.R. observations both for determining positions and for studying the physical nature of comets were made also at the observatories in Alma-Ata, Abastumani, Byurakan, Dushanbe, Kiev (Kiev University and the Academy of Sciences Observatories), and more recently in Pulkovo, Moscow, Odessa and at a number of other observatories.

Astronomers in Kiev have made use of the large telescopes at the observatories in the southern and mountainous regions of the U.S.S.R. As a result, in 1969 over 200 exposures were made for investigating cometary structure and brightness and for determining positions. In the course of this programme the short-period comet 1969 IV Churyumov-Gerasimenko was discovered. 
At the Institute for Theoretical Astronomy ephemerides were calculated for periodic comets Faye and Ashbrook-Jackson (these comets being recovered in 1969 and 1970), as well as for P/Encke and P/Giacobini-Zinner. Systematic calculation of the ephemerides of new comets was organized at ITA in 1965, numerous orbits determined from three and more observations being published in the Kiev Comet Circulars. In these Circulars are published positions reduced with the help of the BESM-4 computer at ITA from the measurements obtained at the Crimean Astrophysical Observatory and at a few other observatories in the U.S.S.R.

Since 1963-1964 the Comet Centre in Kiev has published 105 Comet Circulars that contain, in addition to positions and ephemerides, information about cometary brightness and the various physical processes in comets. They also give short accounts of the results of cometary investigations in the U.S.S.R. In Kiev the absolute magnitudes and other physical features of comets were determined (Vsekhsvyatskij, 1958, 1966a, 1967). Table I shows the values of $H_{10}$ (absolute magnitude), $D_{1}$ (reduced

TABLE I

Absolute magnitudes $\left(\mathrm{H}_{10}\right)$, reduced head diameters $\left(D_{1}\right)$ and maximum tail length $(S)$ for comets 1965-1970

\begin{tabular}{|c|c|c|c|c|}
\hline & Comet & $\mathrm{H}_{10}$ & $D_{1}$ & $S(\mathrm{AU})$ \\
\hline 1965 I & $\mathrm{P} /$ Tsuchinshan 1 & 14.3 & 0.2 & - \\
\hline 1965 II & P/Tsuchinshan 2 & 12.1 & 0.3 & 0.0001 \\
\hline 1965 III & P/Wolf-Harrington & 12.1 & 0.5 & 0.0005 \\
\hline 1965 IV & P/Tempel-Tuttle & 13.6 & - & 一 \\
\hline $1965 \mathrm{~V}$ & P/Reinmuth 1 & 12.1 & - & - \\
\hline $1965 \mathrm{VI}$ & P/Klemola & 13.4 & 0.2 & - \\
\hline 1965 VII & P/de Vico-Swift & 14.4 & 0.3 & 0.0005 \\
\hline 1965 VIII & Ikeya-Seki & 6.2 & 4.2 & 1.3 \\
\hline 1965 IX & Alcock & 9.1 & 0.7 & - \\
\hline 1966 I & P/Giacobini-Zinner & 11.9 & - & - \\
\hline 1966 II & Barbon & 5.5 & 5.9 & 0.33 \\
\hline 1966 III & P/Van Biesbroeck & 10.1 & 0.7 & - \\
\hline 1966 IV & Ikeya-Everhart & 7.5 & 7 & 0.003 \\
\hline $1966 \mathrm{~V}$ & Kilston & 4.5 & 6 & 0.095 \\
\hline 1966 VI & P/Neujmin 1 & 11.0 & 0.4 & \\
\hline 1967 I & P/Grigg-Skjellerup & 14.8 & - & - \\
\hline 1967 II & Rudnicki & $9.7-10.7$ & 2.1 & 0.095 \\
\hline 1967 III & Wild & 10.3 & 2.8 & 0.001 \\
\hline 1967 IV & Seki & 10.5 & 2.0 & 0.001 \\
\hline $1967 \mathrm{~V}$ & P/Tuttle & 10.0 & 2.2 & - \\
\hline 1967 VI & P/Arend & 13.3 & - & - \\
\hline 1967 VII & Mitchell-Jones-Gerber & 5.1 & 13.4 & 0.11 \\
\hline 1967 VIII & P/Borrelly & 12.5 & - & - \\
\hline 1967 IX & P/Finlay & 12.5 & - & - \\
\hline $1967 X$ & P/Tempel 2 & 10.4 & 2 & 0.0005 \\
\hline 1967 XI & P/Reinmuth 2 & 11.7 & 0.8 & - \\
\hline 1967 XII & P/Wolf & 12.6 & - & - \\
\hline 1967 XIII & P/Encke & 11.4 & 1.3 & - \\
\hline 1967 XIV & $\mathrm{P} /$ Wirtanen & 14.3 & 0.1 & $\cdots$ \\
\hline
\end{tabular}


TABLE I (continued)

\begin{tabular}{llccl}
\hline & Comet & $\mathrm{H}_{10}$ & $D_{1}$ & $\mathrm{~S}$ (AU) \\
\hline 1968 I & Ikeya-Seki & 4.4 & 9.5 & 0.045 \\
1968 II & P/Schwassmann-Wachmann & 10.1 & 1.0 & - \\
1968 III & Wild & 7.5 & 1 & 0.004 \\
1968 IV & Tago-Honda-Yamamoto & $10.0-11.0$ & 2.8 & 0.002 \\
1968 V & Whitaker-Thomas & 10.3 & 1.8 & - \\
1968 VI & Honda & 5.5 & $6-9.9$ & 0.041 \\
1968 VII & Bally-Clayton & $7.3-8.0$ & 4.0 & 0.005 \\
1968 VIII & P/Perrine-Mrkos & 15.6 & 0.9 & - \\
1968 IX & Honda & 6.9 & 2.8 & 0.002 \\
\hline 1969 I & Thomas & 5.8 & $2.7-3.8$ & 0.012 \\
1969 II & P/Gunn & 8.9 & - & - \\
1969 III & P/Harrington-Abell & 14.3 & - & - \\
1969 IV & P/Churyumov-Gerasimenko & 10.4 & 1.3 & 0.004 \\
1969 V & P/Honda-Mrkos-Pajdušáková & 11.6 & 1.0 & - \\
1969 VI & P/Faye & 10.8 & - & - \\
1969 VII & Fujikawa & 8.3 & 5.0 & 0.032 \\
1969 VIII & P/Comas Solá & 11.6 & 2 & - \\
1969 IX & Tago-Sato-Kosaka & 6.0 & 7 & 0.220 \\
\hline $1970 \mathrm{a}$ & Daido-Fujikawa & 8.5 & 2 & 0.074 \\
$1969 \mathrm{i}$ & Bennett & 4.0 & 5 & 0.193 \\
$1969 \mathrm{~b}$ & Kohoutek & 7.0 & 2.4 & 0.01 \\
$1970 f$ & White-Ortiz-Bolelli & 6.0 & - & 0.86 \\
$1970 \mathrm{~m}$ & Suzuki-Sato-Seki & 8.7 & 4 & 0.002 \\
$1970 \mathrm{~g}$ & Abe & 4.5 & 5 & 0.15 \\
\hline
\end{tabular}

diameter of the head) and $S$ (maximum tail length) for the comets appearing in 19651970 (Vsekhsvyatskij and Il'ichishina, 1970). These cometographies also record various observations illustrating physical activity in comets.

It has been found that variations in the integral brightness and observations of the plasma tails of comets may give important data about solar activity and conditions in interplanetary space. Study of the data from P/Schwassmann-Wachmann 1 by the method of superposition of epochs has shown that this comet's flares in brightness have a recurrence period of 25-30 days, there being in many cases a direct correspondence between the increases in brightness and geomagnetic disturbances.

During the previous 50 years at least 54 comets showed these flares and other variations in brightness that demonstrate the influence of corpuscular streams in interplanetary space (Vsekhsvyatskij, 1966b). The results indicate that during certain periods the radial streams in the Sun's outer corona reach out even beyond Jupiter's orbit. A great number of cometary brightness curves have been studied in Kiev. The outcome of this investigation is that the variation in brightness may serve to record the dynamical characteristics of the corpuscular field in interplanetary space, not only in the vicinity of the ecliptic plane, but at great distances from it as well.

Despite the considerable frequency of flares and fluctuations in brightness it has 
been found that the integral brightness (i.e., the total brightness of the head) of the overwhelming majority of comets depends on the mean law $y=10$ with $y=2.5 n$ conforming to the empirical law $I=I_{0}{ }^{-n} \Delta^{-2}$. Both direct analysis of photometric curves and study of the distribution of the absolute magnitudes $\mathrm{H}_{10}$ lead to $y=10$ as the most probable value. The question of the changes in the brightness of cometary nuclei is not so clear. Great variations in the luminosity of nuclei (central condensations) may depend on a comet's icy structure and chemical composition (or cometary 'snow') and probably to a great extent on the conditions in interplanetary space (i.e., on the density and velocity of particles in corpuscular streams and the solar wind, the strength of magnetic fields in the streams and interplanetary space - a consequence of the solar flare activity - and solar and galactic cosmic radiation). The latter is proven by the clear-cut dependence of flares in the brightness of comets upon the level of solar activity.

The number of individual comets with well determined absolute magnitudes now amounts to 603. Taking into consideration only the first appearances of shortperiod comets we obtain the distribution of the absolute magnitudes $\mathrm{H}_{10}$ for these 603 comets shown in Figure 1. The range of $\mathrm{H}_{10}$ is from -2 or -3 to +16.4 . The mode of the distribution corresponds to the value $\mathrm{H}_{10}=6.5$. Beyond the region $\mathrm{H}_{10}=5$ or 6 one should observe a systematic decrease in the number of intrinsically faint objects as the result of observational selection. By extrapolating the premaximum curve of the distribution (well represented by the expression $n=3.24 \times 1.84^{\mathrm{H}_{10}}$ ) we find that the true number of comets brighter than absolute magnitude 17 that

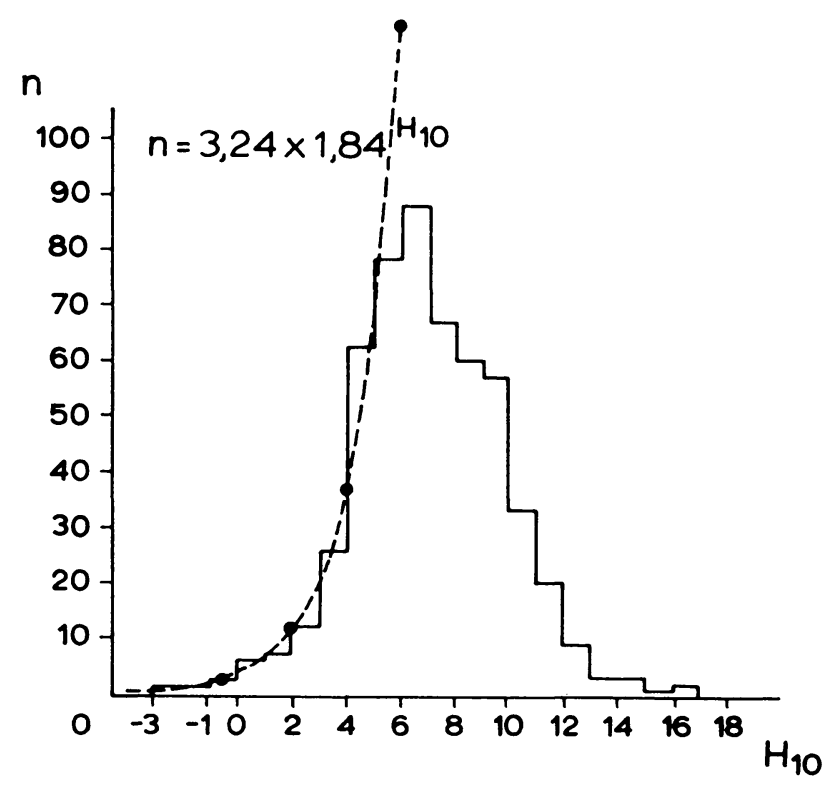

Fig. 1. Distribution of absolute magnitudes of 603 comets (until 1970). Only the first apparitions of periodic comets are included. 
passed through perihelion during 1700-1969 with perihelion distance $q<2$ to $3 \mathrm{AU}$ amounts to $10^{5}$. Thus, the great majority of intrinsically faint comets is unobserved, on account of the apparent faintness of comets of larger perihelion distances, disappearance in twilight, and other conditions of visibility. From this we suppose that the real number of cometary objects should depend on the law $n \sim d^{-k}$ (where $d$ is the comet nucleus diameter, and $k \sim 3$ to 4 from asteroid and meteorite data). The distribution of $\mathrm{H}_{10}$ with $q$ does not show systematic deviations from the mean, and this is an argument which confirms the mean law $y=10$.

Figure 2 shows the distribution of $\mathrm{H}_{10}$ for the first and latest appearances of 102 periodic comets with $P<200$ yr (with 1969 data included). Comets observed at but

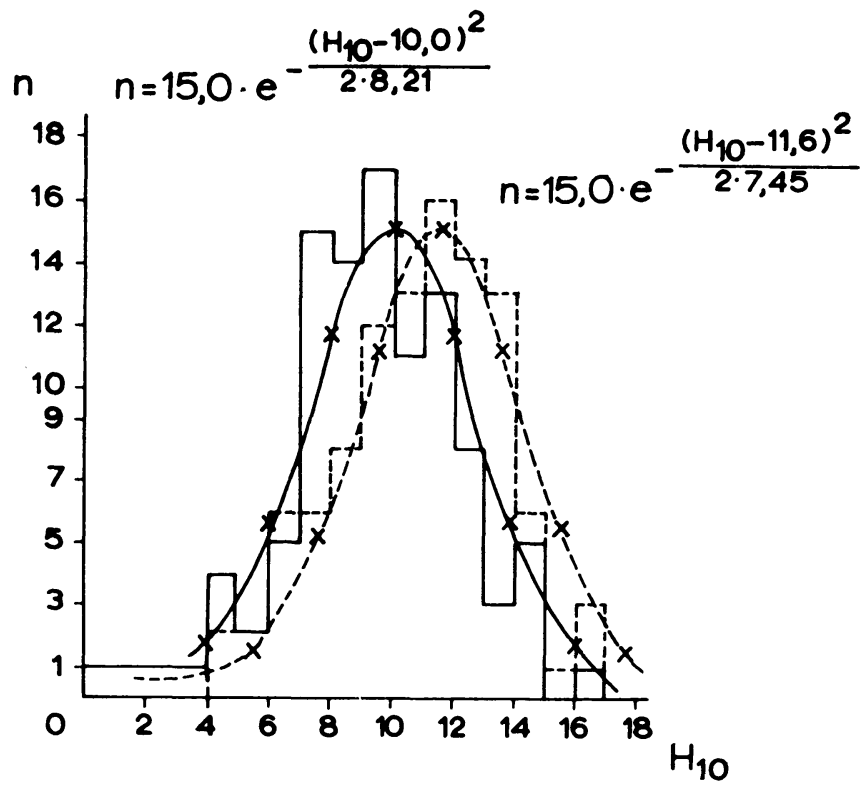

Fig. 2. Comparison of absolute magnitude distribution curves for the first (solid lines) and latest (broken lines) appearances of periodic comets.

one perihelion passage are included in both plots. There is some similarity between the distribution of $\mathrm{H}_{10}$ for short-period comets at their first appearances and the curve for all known comets (i.e., for parabolic and long-period comets). The curve for the latest appearances differs greatly in character and becomes rather asymmetric. The mean epoch for the first appearances is 1877.6 , and that for the latest or single appearances is 1927.8 . The equations for normal distributions that represent the curves are indicated in the figure.

Thus, on the average, during the 50 years the mode has changed from 10.0 to 11.6, indicating a decrease of brightness of 1.6 magnitudes for some mean period of revolution $P=10.9$ yr. This corresponds to a diminution of brightness (i.e., disintegration of comets) of 0.3 to 0.4 magnitude per revolution. The decrease in brightness of individual comets fluctuates from less than 0.1 to 1.0 magnitude per revolution. 
When making statistical investigations of cometary characteristics one should keep in mind, not only the conditions of visibility (i.e., observational selection), but also the recent improvement in observational techniques, efficient sky patrols and the possibility now of observing very faint comets.

In order to make reliable determinations of integral and nuclear magnitudes it is necessary to standardize observations carefully. Even nowadays observers often estimate the brightness only approximately and do not indicate whether they are referring to the head, central condensation or nucleus. Because of this divergences, amounting to 2, 3, or even 4 magnitudes, arise in the estimates by different observers.

Of no less significance can be real effects of comet flare activity, which can appear both in the integral brightness and in the brightness of the central condensation. By uncritical use of the observational material some investigators have acquired a sceptical attitude to the study of cometary activity and the systematic decrease in cometary brightness. It is necessary to set up standard procedures for the calibration of photographic observations of the brightness of comets and systematically to conduct photoelectric and image-tube photometric observations also.

The results must be free from all biases that investigators (who in most cases are not those who make the observations) may have about the systematic diminution of brightness of periodic comets. As we have already stated, the last effect is sometimes masked by real and large fluctuations in brightness.

The question of the systematic decrease in brightness of periodic comets is of very great importance. The data gathered over historic times prove the most important proposition of cometary astronomy: that comets disintegrate rapidly, and that in centuries, if not in decades, short-period comets become inaccessible to observation, not only because of changes in their orbits, but more from the exhaustion of their supplies of cometary ices. It is necessary to study the changes in the orbits of shortperiod comets in order to find out the secular variations of their physical properties. In this sense historical studies - analysis of observations of the past - are to be recommended.

On the other hand, establishment of a cometary observation service today requires definite standardization, not only of the methods of determination of positions, but also of the ways of determining the physical parameters of comets. The first attempts of such a type were made in connection with the IQSY, when comet observations were included in the international programme. This work seems to be continuing.

To determine the integral brightness of comets, photoelectric devices on powerful telescopes, as well as photographic systems (astrographs, Schmidt and Maksutov telescopes), should be used to survey standard areas of stars or regions containing galaxies or planetary nebulae. When publishing the observations mention should be made of the region of the spectrum to which the estimates refer. The procedure used by Roemer in Flagstaff and more recently in Tucson should be followed when measuring the brightness of cometary nuclei with large telescopes.

For comets that are brighter than magnitude 11 or 12 it is necessary to continue making visual (extrafocal) brightness estimates using both wide-field instruments (comet-seekers, binoculars) and refractors of aperture $150-250 \mathrm{~mm}$, so that com- 
parison can be made with turn-of-the-century observations in the so-called Holetschek system.

Despite the considerable success of the international cometary service, particularly in recent years, we cannot consider the present system of cometary observations and discoveries to be perfect. Because of the insufficient number of precise positions, especially of faint comets, we are not able to determine orbits with accuracy sufficient to clarify the true role and nature of nongravitational forces and to study the peculiarities of individual comets as indicators of conditions in interplanetary space.

It is clear that a number of measures should be undertaken to increase activity in searching for new comets. Approaches should be made to individual observatories and societies of amateur astronomers in order to develop visual comet hunting in different countries (particularly in the Southern Hemisphere) and to inspire success such as that recently in Japan and during the late 1940's and 1950's in Czechoslovakia. On the other hand it is necessary to call to the attention of all comet observers the desirability of accompanying their publication of the positions of a comet with a short description of the comet's characteristics (the integral brightness, brightness of central condensation, head diameter, presence and length of tail, its structure, etc.). At present, important information about cometary phenomena often remains lost in the observers' notebooks.

\section{References}

Vsekhsvyatskij, S. K.: 1958, Fizicheskie Kharakteristiki Komet, Moscow. (English translation: 1964, Physical Characteristics of Comets, Jerusalem.)

Vsekhsvyatskij, S. K.: 1966a, Komety 1954-1960, Moscow.

Vsekhsvyatskij, S. K.: 1966b, Mem. Soc. Roy. Sci. Liège Ser. 5 12, 57.

Vsekhsvyatskij, S. K.: 1967, Komety 1961-1965, Moscow.

Vsekhsvyatskij, S. K. and Il'ichishina, N. I.: 1970, Komety 1965-1969 (in press). 